\title{
Avaliação da motivação intrínseca na aprendizagem: validação de duas escalas para crianças e adolescentes
}

\author{
Francisco Simões ${ }^{-}$- Universidade de Coimbra, Coimbra, Portugal \\ Madalena Alarcão - Universidade de Coimbra, Coimbra, Portugal
}

\begin{abstract}
Resumo
O objetivo do presente trabalho é proceder à adaptação e validação de duas escalas breves de avaliação da motivação na aprendizagem: a Escala de Escolha Percebida na Aprendizagem e a Escala de Competência Percebida na Aprendizagem. O estudo abrangeu 510 participantes, com idades compreendidas entre os 9 e os 16 anos. Após a análise exploratória de dados, o trabalho envolveu a avaliação da consistência interna, sucedida de uma análise fatorial exploratória de ambas as escalas. Foram estudadas, também, a sua validade convergente, bem como a sua estabilidade temporal. Foi ainda realizada uma análise diferencial dos resultados quanto às variáveis gênero e ano de escolaridade. Os instrumentos revelam valores moderados a elevados no nível da consistência interna e estruturas unidimensionais à semelhança das versões originais em língua inglesa. Confirmam-se, também, várias das hipóteses colocadas quanto à sua validade convergente. Estudos adicionais relativamente à estabilidade temporal desses instrumentos parecem ser pertinentes.

Palavras-chave: Motivação, Escolha percebida, Competência percebida.
\end{abstract}

Assessment of intrinsic motivation for learning: validation of two scales for children and adolescents

\begin{abstract}
The aim of this study was to adapt and validate two brief scales in order to assess the subjective motivation for learning: the Perceived Choice for Learning Scale and the Perceived Competence for Learning Scale. The study involved 510 participants, ages 9-16 years old. After an exploratory data analysis, the study involved the assessment of internal consistency, followed by an exploratory factor analysis of both scales. Convergent validity and temporal stability of the two scales were also tested, along with a differential analysis regarding gender and school level of the participants. The instruments showed moderate to high levels of internal consistency and one-dimensional structures similar to their original versions in English. Several hypotheses concerning convergent validity were also supported by the study. Additional research seems to be recommendable to address temporal stability issues.

Keywords: Motivation, Perceived choice, Perceived competence.
\end{abstract}

Nas últimas décadas, a análise da motivação em vários contextos, incluindo o da aprendizagem, tem merecido uma atenção crescente por parte de diversas abordagens teóricas. Entre elas merece relevo a Teoria da Autodeterminação (TAD). O seu princípio fundamental é de que cada sujeito possui uma organização motivacional intrínseca orientada para o desenvolvimento pessoal, a integração do Eu e a resolução de inconsistências internas. À luz dessa teoria, a motivação intrínseca descreve os comportamentos que são desenvolvidos com base no interesse e no prazer genuíno que lhes é inerente. Pelo contrário, a motivação extrínseca é definida pela TAD como o processo motivacional em que os comportamentos são condicionados por fatores internos (e.g. estratégias defensivas de confirmação do valor pessoal) ou externos (e.g. recompensas) que levam à operacionalização de ações que não resultam em interesse ou prazer para o sujeito (Vanteenkiste, Lens \& Deci, 2006).

Desenvolvimentos mais recentes da TAD vieram mostrar que a dicotomia entre motivação

\footnotetext{
${ }^{1}$ Endereço para correspondência:

Canada da Francesa, 28, São Mateus da Calheta, Angra do

Heroísmo - 9700-551 - Açores - Portugal

E-mail: francisco.simoes@cipp-terceira.com
}

intrínseca e motivação extrínseca é relevante, mas insuficiente para captar todo o dinamismo inerente aos processos motivacionais. Aspetos como a flutuação das motivações subjetivas ao longo do tempo, o seu condicionamento por via do desenvolvimento pessoal associado ao ciclo de vida e, sobretudo, a evidência crescente de que a própria motivação extrínseca denota variações em função do tipo de regulação e do respectivo grau de autonomia percebida pressionaram a emergência de novas sistematizações teóricas. No sentido de dar uma resposta a estas e a outras exigências, a TAD evoluiu para a definição de um contínuo motivacional da autodeterminação (Ryan e Deci, 2000). Entre outras inovações, esta concepção teórica: a) descreve a motivação como um contínuo bidireccional que vai do comportamento indeterminado, associado à desmotivação, ao comportamento autodeterminado, ligado à motivação intrínseca, passando pelo comportamento determinado externamente que carateriza a motivação extrínseca; b) detalha os estilos de regulação, locus de controle percebido e processos de regulação inerentes a cada um dos campos comportamentais e motivacionais desse mesmo contínuo; c) descreve a motivação extrínseca de forma mais pormenorizada, no que diz respeito aos processos de regulação que lhe estão subjacentes. Em concreto, os autores postulam que a motivação 
extrínseca pode assentar em uma regulação externa ou em mecanismos de introjeção, identificação ou integração.

O modelo do contínuo motivacional vem ainda reforçar a ideia de que a motivação intrínseca é, em primeiro lugar, influenciada pela noção de competência pessoal, associada a um sentido de escolha livre das tarefas em contextos como o das aprendizagens formais (Zisimopoulos \& Galanaki, 2009). Estabelecese, assim, que a realização de uma tarefa de aprendizagem em meio escolar será tanto mais baseada no interesse genuíno e no prazer quanto mais o aluno perceber que é capaz de realizá-la com proficiência, ao mesmo tempo que a compreende como resultado de uma escolha congruente com as suas necessidades e interesses (Deci, Eghrani, Patrick \& Leone, 1994). Por outras palavras, competência e escolha percebidas são as duas variáveis nucleares da motivação intrínseca na aprendizagem, apesar de outras, como a existência de feedback positivo ou a disponibilização de um racional para a realização de tarefas, parecerem também contribuir, embora com pesos e orientações diversas, para a sua definição (Deci e Cols.., 1994; Reeve e Cols., 2003).

Uma maior sistematização teórica dos processos motivacionais por parte da TAD tem sido acompanhada pela construção de alguns instrumentos de avaliação, como questionários e escalas. Alguns deles permitem obter medidas gerais da motivação subjetiva, em diferentes esferas do funcionamento pessoal e social (trabalho, educação, saúde, entre outras). Em outros casos têm sido desenvolvidas escalas de caráter breve, no sentido de avaliar algumas variáveis específicas envolvidas nos processos motivacionais, sendo bastante indicadas para certas populações, de que são exemplo as crianças e os adolescentes (Moreira, 2004).

O estudo vertente posiciona-se nesta segunda linha de avaliação da motivação intrínseca. Deste modo, o seu objetivo geral é o de disponibilizar, em Língua Portuguesa, escalas que permitam avaliar a escolha e a competência percebidas na aprendizagem, em crianças e adolescentes com idades compreendidas entre os 9 e os 16 anos. As mesmas basearam-se na relevância da escolha e da competência percebidas sugerida pela TAD enquanto núcleo definidor da motivação intrínseca. Os resultados deste trabalho, bem como as limitações encontradas e os procedimentos adotados com vista a ultrapassá-las, são apresentados e discutidos nos pontos seguintes.

\section{Método}

Participantes
A amostra deste estudo foi recolhida em cinco escolas da rede de ensino público, da Região Autónoma dos Açores, em Portugal. Foram convidados a participar todos os alunos que frequentavam o ensino regular do $5^{\circ}$ e do $7^{\circ}$ anos de escolaridade, nas escolas envolvidas. Considerando os critérios de inclusão na amostra, foram seleccionadas 27 turmas, com um total de 588 alunos inscritos. A amostra final ficou constituída por 510 alunos, resultando em uma taxa de participação de $86,7 \%$. Destes, 238 (46,7\%) eram do sexo masculino e 272 $(53,3 \%)$ eram do sexo feminino. Em relação ao nível de escolaridade, 246 dos participantes frequentavam o $5^{\circ}$ ano, ao passo que 264 estavam inseridos em turmas do $7^{\circ}$ ano de escolaridade. A idade dos participantes variou entre os 9 e os 16 anos $(M=11,12 ; D P=1,24)$.

\section{Instrumentos}

Escala de Escolha Percebida na Aprendizagem

A Escala de Escolha Percebida na Aprendizagem é uma subescala da Escala de Autodeterminação (Sheldon, 1995; Trash \& Elliot, 2002). A mesma foi adaptada a partir da versão traduzida para a população portuguesa (Silva e Cols., 2007). Esta escala avalia em que medida os sujeitos consideram as suas decisões e escolhas na aprendizagem como resultado da sua própria iniciativa e é constituída por 5 itens formulados de modo afirmativo (e.g. Sinto que sou a escolher aquilo que faço). Em relação a cada uma das afirmações, os sujeitos deverão posicionar-se de acordo com uma escala ordinal de 1 (nunca) a 5 (sempre). Diversos estudos realizados com a versão original revelam valores de alfa de Cronbach que variam entre 0,86 e 0,92 (Sheldon, 1995). A versão, para adultos, adaptada para a população portuguesa apresenta um alfa de Cronbach de 0,85 (Silva e Cols., 2007).

\section{Escala de Competência Percebida na Aprendizagem}

A Escala de Competência Percebida na Aprendizagem é constituída por quatro itens que avaliam o grau subjetivo de competência dos sujeitos, em um determinado contexto de aprendizagem. O instrumento, na sua versão original, em Língua Inglesa, permite ao sujeito analisar o seu grau de concordância em relação a cada uma das afirmações, de acordo com uma escala de resposta de sete pontos (de completamente falso a completamente verdadeiro). $\mathrm{Na}$ sua versão adaptada para crianças e adolescentes da população Portuguesa, cada um dos itens (e.g. Vou ser capaz de atingir os meus objetivos, neste ano letivo) é avaliado por meio do recurso a uma escala de ordinal de cinco pontos, que varia entre 1 (nunca) e 5 (sempre). Não são 
conhecidos estudos sobre as qualidades psicométricas desta escala.

\section{Escala de Satisfação das Necessidades Psicológicas Básicas na Relação}

A Escala de Satisfação das Necessidades Psicológicas Básicas na Relação (ESNPBR) é constituída por um conjunto de nove itens, que podem ser classificados de 1 (nunca) a 5 (sempre). Eles estão agrupados em três fatores, que correspondem ao suporte das necessidades psicológicas básicas definidas pela TAD (relação, competência e autonomia). Os três itens em cada um dos fatores estão formulados de modo afirmativo, um deles sendo cotado em sentido inverso, pois nos seus níveis mais elevados descreve uma frustração da necessidade psicológica básica a que se reporta. A sua administração permite que $\mathrm{o}$ aplicador selecione aquela relação sobre a qual pretende fazer a avaliação da satisfação das necessidades psicológicas básicas, possibilitando a avaliação de diferentes tipos de relacionamentos pessoais (La Guardia, Ryan, Couchman \& Deci, 2000). Na sua versão original, a ESNPBR revelou valores de alfa de Cronbach entre 0,85 e 0,94 (La Guardia e Cols., 2000). A versão Portuguesa deste instrumento, adaptada para crianças e adolescentes e destinada a avaliar relações pedagógicas, revelou níveis de consistência interna moderados a elevados $(0,80)$ e um coeficiente de validade teste-reteste de 0,58 (Simões \& Alarcão, no prelo).

\section{Escala de Esperança para Crianças}

A Escala de Esperança para Crianças (EEC) foi desenvolvida por Snyder e Cols. (1997) para avaliar os pensamentos de esperança relacionados com objetivos, em crianças e adolescentes com idades entre os 8 e os 16 anos. A escala é constituída por 6 itens, formulados de modo afirmativo, sendo que três deles avaliam o componente denominado "Iniciativa" (e.g. Penso que estou a fazer bem as coisas) e os restantes três itens avaliam o componente designado por "Caminhos" (e.g. Consigo pensar em muitas maneiras de conseguir as coisas que considero importantes). Os itens que avaliam cada um dos componentes são apresentados de uma forma alternada e podem ser classificados de acordo com uma escala ordinal de seis pontos entre 1 (nenhuma das vezes) e 6 (todas as vezes). Valores mais elevados correspondem a níveis mais significativos de esperança. Estudos realizados pelos autores da versão original revelam consistência interna com valores que variaram entre 0,72 e 0,86 e um coeficiente de fidelidade teste-reteste com valores que oscilam entre 0,71 e 0,73 (Snyder e Cols., 1997). Um alfa de Cronbach de 0,81 foi encontrado para a versão portuguesa, assim como um coeficente de fidelidade teste-reteste de 0,60 (Marques, Pais-Ribeiro \& Lopez, 2009).

\section{Procedimentos}

Adaptação dos instrumentos para a Lingua Portuguesa

A realização do estudo foi antecedida de um pedido de autorização aos autores para utilização e adaptação dos instrumentos. No caso da Escala de Escolha Percebida na Aprendizagem, estando já disponível uma versão em Língua Portuguesa destinada a adultos, foram realizados os ajustamentos necessários ao enquadramento do instrumento no contexto da aprendizagem formal, bem como à adequação da linguagem ao nível etário dos sujeitos. A alteração mais relevante foi o abandono da estrutura dicotómica dos itens, constituída por duas afirmações contraditórias: uma colocada no primeiro ponto da escala de resposta e a outra no extremo oposto desse contínuo. Em vez dessa organização, cada item passou a ser constituído por apenas uma afirmação em relação à qual os sujeitos teriam de posicionar-se, de acordo com a escala de resposta fornecida.

Por sua vez, a Escala de Competência Percebida na Aprendizagem foi traduzida da sua versão original em inglês para a língua portuguesa por duas pessoas formadas em psicologia e fluentes em ambos os idiomas. Seguiu-se um processo de retradução, realizado por dois docentes formados nas duas línguas, sem acesso à versão original do instrumento, no intuito à favorecer a identificação de discrepâncias entre ambas as formulações. De maneira a garantir um equilíbrio entre as definições teóricas das necessidades psicológicas básicas, tal como são propostas na literatura pela TAD, e a preocupação de tornar a linguagem acessível a crianças e adolescentes, o processo de tradução e retradução foi triangulado com a opinião de outros dois docentes do ensino básico com formação superior em português e inglês.

Após o processo de tradução/retradução da Escala de Escolha Percebida na Aprendizagem e da adaptação da Escala de Competência Percebida na Aprendizagem, foi realizado um estudo piloto. Nele participaram 15 crianças e adolescentes, oito do sexo feminino e sete do sexo masculino, com idades compreendidas entre os 10 e os 16 anos $(M=11,28$; $D P=2,12)$. Todos frequentavam o $5^{\circ}$ ou o $7^{\circ}$ ano de escolaridade e dois projetos locais de ocupação de tempos livres conhecidos pelos investigadores. A sua seleção foi feita em função de três critérios: a) serem alunos das escolas abrangidas pelo estudo; b) estarem dentro da faixa etária esperada para a amostra; c) serem alunos com um nível de literacia inferior ao esperado para a idade, de modo a melhor analisar as questões de compreensão dos itens propostos (Moreira, 2004). O 
consentimento informado dos responsáveis legais das crianças e adolescentes que participaram no estudo piloto foi recolhido através de documento escrito criado para o efeito. Nele eram descritos os objetivos e procedimentos do estudo a realizar, ao mesmo tempo que se solicitava a autorização para que os respectivos educandos nele participassem. No momento da aplicação dos instrumentos, os participantes foram sempre questionados sobre a sua disponibilidade para colaborarem no estudo, mesmo após ter sido obtido o consentimento informado dos respectivos responsáveis legais, sendo do mesmo modo garantida a confidencialidade dos dados fornecidos. Os que aceitaram participar fizeram o preenchimento individual dos instrumentos e, em seguida, discutiram o processo de resposta com o primeiro autor deste estudo, presente durante a administração. A triangulação da informação com docentes, bem como a discussão item a item realizada com os sujeitos que participaram no estudo piloto, conduziu a ajustamentos em três itens da Escala de Escolha Percebida na Aprendizagem. As alterações introduzidas foram feitas no nível da sintaxe dos itens, substituição de palavras por sinónimos mais inteligíveis ou emprego de diferentes tempos verbais.

\section{Amostragem}

Depois de concluído o processo de adaptação dos instrumentos, foi solicitada autorização à Direcção Regional da Educação e Formação do Governo Regional dos Açores para a administração do protocolo do estudo em escolas da rede pública de ensino da região. Obtido o consentimento oficial da tutela, foi feito um pedido de colaboração a cinco escolas, mediante contato com os respectivos conselhos executivos. Estas foram selecionadas considerando que já colaboravam com os investigadores em outro estudo sobre o impacto de um programa de mentoria escolar, tendo todos mostrado disponibilidade para participar na investigação. Em sequência, foi garantido o consentimento informado dos responsáveis legais pelas crianças e adolescentes das turmas abrangidas, através de documento escrito enviado pelo diretor de turma semelhante ao que já fora usado no estudo piloto. No momento de aplicação, além de serem elucidados sobre os objetivos do estudo, as questões de confidencialidade e o modo de preenchimento dos instrumentos, os participantes foram de novo questionados sobre a sua vontade para colaborarem no estudo.

A aplicação dos instrumentos foi coletiva, tendo sido realizada em aulas da disciplina de Cidadania, de acordo com o estipulado pelos conselhos executivos das escolas e ouvidos os diretores de turma quanto à sua disponibilidade. A dimensão dos grupos variou entre um mínimo de 10 e um máximo de 25 alunos. A administração dos instrumentos foi feita pelo primeiro autor, de acordo com um protocolo de procedimentos criado para o efeito, não estando presente qualquer docente na sala no momento da aplicação. Antes de preencherem os instrumentos, os participantes foram informados sobre o modo de organização do protocolo, as questões de confidencialidade das respostas e a importância de todas as perguntas serem respondidas.

\section{Análise dos dados}

Os dados foram analisados com recurso ao PASW Statistics 18. Primeiro, foi realizada uma avaliação da consistência interna dos instrumentos, através do cálculo do alfa de Cronbach para a totalidade da escala e da análise deste parâmetro na contingência de eliminação de cada um dos itens (scale if item-deleted) que o constituem. De seguida, foi feita uma Análise em Componentes Principais. Os componentes retidos foram aqueles que apresentavam um eigenvalue superior a 1 , em consonância com o scree plot e a percentagem de variância retida (Maroco, 2007). Este procedimento foi sucedido de uma análise fatorial confirmatória realizada no programa Amos 18 e que se destinou a aumentar a compreensibilidade do modelo. Seguiu-se uma análise da validade convergente, mediante o cálculo de um conjunto de correlações de Spearman entre todas as variáveis dos diferentes questionários e escalas integrados na bateria deste estudo. Foi conduzido, também, um estudo diferencial dos resultados, comparando-os com base nas variáveis gênero e ano de escolaridade, se aplicando, para tal, o teste $t$ de Student para amostras independentes. Por último, foi verificada a estabilidade temporal das respostas, através de uma segunda aplicação de toda a bateria de instrumentos. O reteste envolveu um número bastante menor de participantes devido a períodos de interrupção letiva que ocorrem todos os anos nas escolas para efeitos de formação profissional obrigatória dos professores. Uma vez que este estudo se integrava em um projeto de investigação mais vasto, não foi possível alargar o período temporal do reteste, de modo a melhorar as condições de constituição da amostra, no que diz respeito ao número de sujeitos envolvidos. A avaliação da estabilidade temporal do instrumento foi feita recorrendo ao cálculo do coeficiente de correlação de Spearman entre ambos os momentos de aplicação, os quais distaram duas semanas entre si.

\section{Resultados}




\section{Consistência interna}

A análise da consistência interna revelou valores de alfa de Cronbach de, respectivamente, 0,80 e 0,85, para a Escala de Escolha Percebida na Aprendizagem e para a Escala de Competência Percebida na Aprendizagem, os quais podem ser considerados moderados a elevados (Murphy \& Davidshofer, 1988). Procedeu-se, de igual modo, a uma análise da consistência interna das duas escalas, com base no critério scale if item-deleted. Em ambos os casos, a remoção de qualquer um dos itens que constituíam os instrumentos analisados não resultou em um acréscimo do valor global dos valores respectivos de alfa de Cronbach, mas antes na sua diminuição.

Análise em Componentes Principais

\section{Escala de Escolha Percebida na Aprendiragem}

A Análise em Componentes Principais, no caso da Escala de Escolha Percebida na Aprendizagem, revelou um valor de $\mathrm{KMO}=0,740$, indicando uma fatoriabilidade média. Todas as correlações entre os itens encontravam-se acima de 0,30. A Tabela 1 resume a estrutura fatorial encontrada.

Tabela 1. Escala de Escolha Percebida na Aprendizagem (Loadings, Eigenvalue e Percentagem de Variância Explicada)

\begin{tabular}{ccc}
\hline $\mathrm{N}^{\circ}$ item & Item & Componente 1 \\
\hline 1 & Sinto que sou eu a escolher aquilo que faço. & 0,687 \\
2 & Faço as coisas que tenho a fazer por escolha própria. & 0,745 \\
3 & Eu faço aquilo que faço, porque me interessa. & 0,687 \\
4 & Sinto-me livre para fazer as coisas que decido fazer. & 0,812 \\
5 & Sinto-me livre para fazer aquilo que escolho fazer. & 0,800 \\
\hline Eigenvalue & & 2,799 \\
$\%$ de variância explicada & & 55,97 \\
\hline
\end{tabular}

A análise em componentes principais mostrou a unidimensionalidade da Escala de Escolha Percebida na Aprendizagem, se identificando um componente responsável por $55,97 \%$ da variância total explicada, com um eigenvalue de 2,799 .

Escala de Competência Percebida na Aprendizagem
No caso da Escala de Competência Percebida na Aprendizagem, a análise em componentes principais apresentou um valor de $\mathrm{KMO}=0,812$, indicando uma boa fatoriabilidade. Todas as correlações inter itens se encontravam acima de 0,50. ATabela 2 apresenta uma síntese dos componentes extraídos.

Tabela 2. Escala de Competência Percebida na Aprendizagem (Loadings, Eigenvalue e Percentagem de Variância Explicada)

\begin{tabular}{ccc}
\hline $\mathrm{N}^{\circ}$ item & Item & Componente 1 \\
\hline 1 & Sinto confiança para aprender a maior parte das & 0,841 \\
2 & matérias. & Sou capaz de aprender a maior parte das matérias. \\
3 & $\begin{array}{c}\text { Vou ser capaz de atingir os meus objectivos, neste ano } \\
\text { lectivo. }\end{array}$ & 0,858 \\
& Vou ser capaz de ter boas notas, na maior parte das \\
& disciplinas. & 0,800 \\
& & 2,759 \\
\hline $\begin{array}{l}\text { Eigenvalue } \\
\text { \% de variância explicada }\end{array}$ & & 68,97 \\
\hline
\end{tabular}

A análise em componentes principais revelou, também, a unidimensionalidade da Escala de Competência Percebida na Aprendizagem, se identificando um componente responsável por $68,97 \%$ da variância total explicada, com um eigenvalue de 2,759 .

Validade convergente
Tal como ilustra a Tabela 3, foi possível observar que as diferentes escalas se encontram significativamente correlacionadas.

Uma leitura mais atenta da Tabela 3 permite verificar que algumas das correlações no nível da validade convergente entre as Escalas de Competência Percebida na Aprendizagem e de Escolha Percebida na Aprendizagem $\mathrm{e}$ as subescalas dos instrumentos 
aplicados são não só significativas, mas também moderadas ( $\rho>0,30)$.

Tabela 3. Correlações entre a Escala de Escolha Percebida na Aprendizagem e a Escala de Competência Percebida na Aprendizagem e os instrumentos incluídos no protocolo administrado

\begin{tabular}{lcc}
\hline Instrumentos & $\begin{array}{c}\text { Escala de Escolha Percebida } \\
\text { na Aprendizagem }\end{array}$ & $\begin{array}{c}\text { Escala de Competência Percebida na } \\
\text { Aprendizagem }\end{array}$ \\
\hline $\begin{array}{l}\text { Escala de Escolha Percebida na } \\
\text { Aprendizagem }\end{array}$ & --- & $0,31^{* *}$ \\
Escala de Competência Percebida & $0,31^{* *}$ & -- \\
na Aprendizagem & & \\
ESNPBR/Suporte para a Relação & $0,11^{* *}$ & $0,32^{* *}$ \\
ESNPBR/Suporte para a Competência & $0,26^{* *}$ & $0,45^{* *}$ \\
ESNPBR/Suporte para a Autonomia & $0,15^{* *}$ & $0,26^{* *}$ \\
Esperança/Caminhos & $0,43^{* *}$ & $0,55^{* *}$ \\
Esperança/Iniciativa & $0,44^{* *}$ & $0,55^{* *}$ \\
\hline
\end{tabular}

Nota. ${ }^{* *} P<0,001$.

Neste capítulo, será de realçar, em primeiro lugar, a correlação moderada entre a Escala de Escolha Percebida na Aprendizagem e a Escala de Competência Percebida na Aprendizagem ( $\varrho>0,31, p<0,01)$. No caso da Escala de Escolha Percebida na Aprendizagem, esta apresenta correlações também moderadas, mas mais elevadas, com as duas subescalas da Escala de Esperança, a subescala Iniciativa $(\varrho s>0,43, p<0,01)$ e a subescala Caminhos ( $\rho s>0,43, p<0,01$ ). De acordo com o mesmo critério, se destacam, também, as correlações moderadas entre a Escala de Competência Percebida na Aprendizagem e as subescalas da Escala de Esperança, a subescala Caminhos $(\varrho s>0,55, p<0,01)$ e a subescala Iniciativa ( $\varrho>0,55, p<0,01)$. É de salientar, ainda, as correlações moderadas entre a Escala de Competência Percebida na Aprendizagem e a subescalas Suporte para a Competência ( $\rho>0,45$, $p<0,01)$ e Suporte para a Relação ( $\rho>0,32, p<0,01)$. De notar que quer a Escala de Escolha Percebida na Aprendizagem, quer a Escala de Competência Percebida na Aprendizagem apresentam correlações positivas e significativas, mas fracas, com a subescala de Suporte para a Autonomia, da ESNPBR, ao invés do que era previsto antes da realização deste estudo.

\section{Análise diferencial}

A análise diferencial revelou diferenças significativas entre alunos do $5^{\circ}$ e do $7^{\circ}$ ano de escolaridade no que se refere à competência percebida na aprendizagem $[t(508)=8,154 ; p=0,05)]$. Os alunos do $5^{\circ}$ ano de escolaridade apresentaram um resultado médio superior $(M=17,32 ; \mathrm{DP}=2,50)$ em comparação com os alunos do $7^{\circ}$ ano $(M=15,54 ; \mathrm{DP}=2,41)$. Não foram, contudo, detetadas diferenças estatisticamente significativas quanto à escolha percebida na aprendizagem, tendo em conta a variável ano de escolaridade. Quando é considerada a variável gênero, os resultados obtidos por rapazes e raparigas não se diferenciaram do ponto de vista estatístico.

\section{Estabilidade temporal}

Dos 221 alunos envolvidos no reteste, 118 eram do sexo masculino e 103 do sexo feminino, com idades que oscilavam entre os 9 e os 16 anos $(M=11,71$; $D P=1,21)$. No que se refere ao nível de escolaridade, 48 destes alunos estavam matriculados no $5^{\circ}$ ano, enquanto 173 estavam matriculados no $7^{\circ}$ ano. $\mathrm{O}$ valor encontrado para a estabilidade temporal da Escala de Escolha Percebida na Aprendizagem foi moderado ( $\varrho s>0,50, p<0,01$ ), ao passo que para a Escala de Competência Percebida na Aprendizagem foi forte ( $\rho s>0,73, p<0,01)$.

\section{Discussão}

A TAD define a motivação intrínseca como o processo em que o sujeito executa uma dada tarefa pelo interesse genuíno e pelo prazer que lhe são inerentes (Deci e Cols., 1994). As suas dimensões nucleares são a escolha e a competência percebidas na tarefa (Zisimopoulos \& Galanaki, 2009).

No intuito de avaliar com maior propriedade os processos motivacionais, a TAD tem favorecido o desenvolvimento de medidas gerais da motivação intrínseca e de instrumentos de menor extensão que avaliam as suas dimensões particulares. Considerando importante disponibilizar, em Língua Portuguesa, instrumentos breves baseados nos princípios da TAD e ajustados às caraterísticas de crianças e adolescentes (Moreira, 2004), o objetivo fundamental do presente trabalho foi o de analisar as qualidades psicométricas da Escala de Escolha Percebida na Aprendizagem e da Escala de Competência Percebida na Aprendizagem. 
Ambos os instrumentos revelam valores de consistência interna moderados a elevados, com um alfa de Cronbach de, respectivamente, 0,80 e 0,85 . A análise deste parâmetro com base no critério if item deleted parece indiciar uma contribuição equilibrada e relevante dos diferentes itens para a consistência interna dos instrumentos.

A Análise em Componentes Principais evidenciou a unidimensionalidade de ambas as escalas. Todavia, a Escala de Competência Percebida na Aprendizagem apresenta uma melhor fatoriabilidade $(\mathrm{KMO}=0,812)$, ao mesmo tempo que o componente encontrado explica uma maior percentagem de variância explicada $(68,97 \%)$, comparativamente ao que sucede com a Escala de Escolha Percebida na Aprendizagem. Neste caso, a fatoriabilidade é apenas média $(\mathrm{KMO}=0,740)$ e a percentagem da variância explicada pelo componente encontrado é, também, menor $(55,97 \%)$.

O estudo da validade convergente demonstra que todas as escalas e subescalas dos instrumentos usados se encontram positiva e significativamente correlacionadas entre si, com algumas das correlações a apresentarem valores moderados $(\varrho s>0,30)$. Salientamse as correlações entre a Escala de Competência Percebida e as subescalas Iniciativa $(\varrho s>0,55)$ e Caminhos ( $\varrho>0,55)$, da Escala de Esperança para Crianças. A Escala de Escolha Percebida na Aprendizagem e a Escala de Competência Percebida na Aprendizagem apresentam, por sua vez, uma correlação moderada entre si $(\varrho s>0,31)$.

Do ponto de vista do comportamento diferencial dos resultados, considerando as variáveis gênero e ano de escolaridade, verifica-se que apenas no nível da comparação entre alunos de $5^{\circ}$ e $7^{\circ}$ anos de escolaridade se encontrou uma diferença significativa [ $t$ (508) $=8,154 ; p=0,05)$, com os alunos do $5^{\circ}$ ano a apresentarem resultados médios mais elevados na competência percebida. Quanto à avaliação da estabilidade temporal, a Escala de Competência Percebida na Aprendizagem apresenta uma correlação forte entre as duas aplicações efetuadas ( $\rho>0,73)$, ao passo que a Escala de Escolha Percebida na Aprendizagem apresenta uma estabilidade temporal moderada $(\varrho s>0,50)$.

Os resultados obtidos quer no estudo da validade convergente, quer no que se refere à estabilidade temporal de ambas as escalas estão em linha com o conhecimento produzido em estudos anteriores e com as hipóteses colocadas pelos autores do presente estudo. Registra-se, por exemplo, uma associação positiva, significativa e moderada entre as duas escalas avaliadas, bem como entre estas e as subescalas de Suporte para a Relação e de Suporte para a Competência da ESNPBR. De modo complementar, observam-se correlações do mesmo tipo entre as duas escalas estudadas e as subescalas da Escala de Esperança para Crianças. Também os valores para a estabilidade temporal são compatíveis com as hipóteses avançadas, ficando, todavia, aquém do esperado em termos de magnitude para aplicações separadas entre si por apenas duas semanas ( $\varrho>>0,80$ ) (Moreira, 2004). Algumas interpretações podem ser avançadas, no sentido de melhor compreender estes resultados, sobretudo, aqueles mais inesperados. Por um lado, as caraterísticas dos sujeitos poderão ter levado a algum acaso nas respostas, frequente nesta faixa etária, e a algumas dificuldades de compreensão, apesar de todos os procedimentos adotados de modo a preveni-las. No entanto, aspetos inerentes ao desenvolvimento dos sujeitos poderão ser mais esclarecedores. Em primeiro lugar, as correlações obtidas entre as escalas analisadas e a subescala de Suporte para a Autonomia da ESNPBR foram fracas, apesar do cuidado em selecionar instrumentos para testar a validade convergente que partissem do mesmo referencial teórico e que se reportassem ao contexto de aprendizagem. Em segundo lugar, a estabilidade temporal da Escala de Escolha Percebida na Aprendizagem é menor em comparação com a estabilidade temporal evidenciada pela Escala de Competência Percebida na Aprendizagem. Será prudente analisar estes dados ponderando a capacidade dos participantes em avaliar dimensões ligadas à autonomia, como acontece com a Escala de Escolha Percebida na Aprendizagem, em especial quando a avaliação subjetiva sobre esta variável é enquadrada na relação com terceiros. Sendo uma fase marcada por contínuos reajustamentos, desenvolvimentos rápidos e pelo conflito permanente entre a necessidade de afirmação pessoal e a definição de limites pelos adultos, tal poderá resultar em flutuações importantes na apreciação destas dimensões ou torná-la dependente dos contextos ou das pessoas perante os quais ela se define (Liang, Spencer, Brogan \& Corral, 2008). Nesta linha de pensamento, convém realçar o fato da subescala Suporte para a Autonomia, da ESNPBR, reportar-se, neste estudo, à avaliação do aluno sobre a relação com o diretor de turma. Sucede que, no sistema de ensino português, o diretor de turma acaba por estar, muitas vezes, implicado no controle de aspetos como a assiduidade ou a indisciplina, minando ou colocando em causa estratégias baseadas na escolha livre ou na promoção da iniciativa. Tal corrobora, em certa medida, o carácter contingencial das avaliações que poderão ter sido feitas sobre o suporte para a autonomia disponibilizado pelos diretores de turma afetando a correlação desta variável com a Escala de 
Escolha Percebida na Aprendizagem. Em linha com esta perspectiva, note-se que, quando os participantes avaliam a sua autonomia em uma lógica independente da relação com figuras adultas, os valores correlacionais voltam a ser moderados, tal como sucede entre a Escala de Escolha Percebida na Aprendizagem e as subescalas Iniciativa e Caminhos, da Escala de Esperança para Crianças.

Os resultados poderão ter sido, também, influenciados por variáveis contextuais. A normalização das condições de aplicação dos instrumentos, por meio da elaboração de um protocolo de procedimentos detalhado, visou responder a este possível desafio. Todavia, a sua administração coletiva, em meio escolar, pode ter produzido alguns enviesamentos. Por um lado, poderá ter prevalecido alguma dificuldade em estabelecer uma distinção clara entre o processo de investigação e as atividades de aprendizagem formal e, por sua vez, entre o próprio investigador e os professores. É, portanto, possível que os sujeitos se tenham tornado mais sensíveis a aspetos como a confidencialidade das suas respostas. Do mesmo modo, é provável que a massificação de processos de investigação por questionário em meio escolar, em Portugal, tenha condicionado a atenção ou a motivação dos sujeitos, com consequências relevantes na qualidade da sua participação. Este elemento poderá ter sido muito relevante no estudo da estabilidade temporal dos instrumentos, dado que se tratou da repetição de uma tarefa em um espaço de tempo curto (Strange, Forest \& Oakley, 2003). A par dessas dificuldades, a metodologia de recolhimento de dados, ainda no que diz respeito ao estudo da estabilidade temporal, colocou um problema suplementar. Tal residiu no fato do reteste ter sido realizado com os alunos disponíveis para esse efeito e não com um recorte aleatório da amostra ou a sua totalidade. Produziram-se, assim, desequilíbrios, notórios na falta de proporcionalidade entre o número de alunos do $5^{\circ}$ ano e o número de alunos do $7^{\circ}$ ano que preencheram a segunda aplicação, com uma sobrerrepresentação desses últimos.

No caso da análise diferencial dos resultados, esta contradiz, em parte, estudos anteriores que apontam para um incremento da competência percebida na aprendizagem à medida que os alunos vão avançando no seu percurso escolar (Obach, 2003). Este não é, porém, um dado consensual na literatura. Outros trabalhos referem uma diminuição progressiva da motivação intrínseca e dos seus componentes à medida que os alunos progridem, em especial nos anos de mudança de ciclo de estudo, como sucede com os alunos do $7^{\circ}$ ano (Zisimopoulos \& Galanaki, 2009). Já a inexistência de diferenças entre alunos do sexo feminino e do sexo masculino quanto às variáveis em estudo está de acordo com outros resultados conhecidos (Obach, 2003).

\section{Considerações finais}

O presente estudo procurou aferir as qualidades psicométricas da Escala de Escolha Percebida na Aprendizagem, bem como da Escala de Competência Percebida na Aprendizagem. Os dados indicam níveis de consistência interna moderados a elevados, confirmam a unidimensionalidade de ambas as escalas e ainda várias hipóteses colocadas no nível da validade convergente. Dessa forma, considera-se que ficam disponíveis, para utilização em língua portuguesa, duas escalas que permitem avaliar a forma como as crianças e os adolescentes percebem a sua competência e a sua capacidade de escolha na aprendizagem. Estes instrumentos poderão ser úteis em diferentes pesquisas, não só pela sua natureza breve, mas, acima de tudo por avaliarem duas variáveis preditoras da motivação intrínseca em contexto educativo sobre as quais se tem notado um interesse crescente na literatura. Ambas as escalas se beneficiarão da realização de estudos adicionais, sobretudo no nível da sua estabilidade temporal, com diferentes intervalos entre a primeira e a segunda aplicação.

\section{Referências}

Deci, E. L. \& Ryan, R. M. (1985). Intrinsic motivation and self-determination in buman behavior. Nova Iorque: Plenum.

Deci, E. L., Eghrani, H., Patrick, B. C. \& Leone, D. R. (1994). Facilitating internalization: the selfdetermination perspective. Journal of Personality, 62(1), 119-142.

La Guardia, J. G., Ryan, R. M., Couchman, C. E. \& Deci, E. L. (2000). Within-person variation in security of attchament: a self-determination perspective no attachment, need fulfillment, and well-being. Journal of Personality and Social Psychology, 79(3), 367-384.

Liang, B., Spencer, R., Brogan, D. \& Corral, M. (2008). Mentoring relationships from early adolescence through emerging adulthood: a qualitative analysis. Journal of Vocational Behavior, 72, 168-182.

Maroco, J. (2007). Análise estatística com utilização de SPSS. Lisboa: Edições Sílabo.

Marques, S. C., Pais-Ribeiro, J. L. \& Lopez, S. J. (2009). Validation of a portuguese version of the 
Children's Hope Scale. School Psychology International, 30(5), 538-551.

Moreira, J. (2004). Questionários: teoria e prática. Coimbra: Almedina.

Murphy, K. R. \& Davidshofer, C. O. (1988). Psychological testing: principles and applications. Englewood Cliffs, Nova Jersey: Prentice Hall.

Obach, M. S. (2003). A longitudinal-sequential study of perceived academic competence and motivational beliefs for learning among children in middle school. Educational Psychology, 23(3), 323-338.

Reeve, J., Nix, G. \& Hamm, D. (2003). Testing models of experience of self-determination in intrinsic motivation and the conundrum of choice. Journal of Educational Psychology, 95(2), 375-392.

Ryan, R. M. \& Powelson, C. L. (1991). Autonomy and relatedness as fundamental to motivation and education. The Journal of Experimental Education, 34(1), 49-66.

Ryan, R. M. \& Deci, E. L. (2000). Self-determination theory and the facilitation of intrinsic motivation, social development, and well-being. American Psychologist, 55(1), 68-78.

Ryan, R. M., Koestner, R. \& Deci, E. L. (1991). Egoinvolved persistence: when free-choice behavior is not intrinsically motivated. Motivation and Emotion, 15(3), 185-205.

Sheldon, K. M. (1995). Creativity and selfdetermination in personality. Creativity Research Journal, 8(1), 25-36.

Silva, M. N., Vieira, P. N., Minderico, C. S., Castro, M. M., Coutinho, S. C., Santos, T. C. \& Teixeira, P. J. (2007). Testing a portuguese self-determination theory-based psychometric battery. Trabalho apresentado no Annual Meeting of International Society for Behavioral Nutrition and Physical Activity, Oslo.

Simões, F. \& Alarcão, M. (no prelo). Avaliação de satisfação de necessidades psicológicas básicas na relação para a população portuguesa: adaptação e validação da ESNPBR.

Snyder, C. R., Hoza, B., Pelhman, W. E., Rapoff, M., Ware, L., Danovsky, M., Highberger, L., Rubistein, H. \& Stall, K. J. (1997). The development and validation of the Children's Hope Scale. Journal of Pediatric Psychology, 22, 399-421.

Strange, V., Forest, S. \& Oakley A. (2003). Using research questionnaires with young people in schools: the influence of the school context. International Journal of Social Research Methodology, 6(4), 337-346.

Trash, T. M. \& Elliot, A. J. (2002). Implicit and selfattributed achievement motives: concordance and predictive validity. Journal of Personality, 70(5), 729756.

Vanteenkiste, M., Lens, W. \& Deci, E. L. (2006). Intrinsic versus intrinsic goal contents in selfdetermination theory: Another look at the quality of academic motivation. Educational Psychologist, 41(1), 19-31.

Zisimopoulos, D. A. \& Galanaki, E. P. (2009). Academic intrinsic motivation and perceived academic competence in Greek elementary students with and without learning disabilities. Learning Disabilities Research and Practice, 24(1), 3343.

Recebido em 18/04/2011

Reformulado em 05/09/2011

Aprovado em 03/11/2011

Trabalho financiado pela Fundação para a Ciência e Tecnologia do Ministério da Ciência, Tecnologia e Ensino Superior de Portugal (SFRH /BD/60823/2009).

Sobre os autores:

Francisco Simões é doutorando em Psicologia Clínica da Família pela Universidades de Coimbra e de Lisboa. Bolseiro de investigação da Fundação para a Ciência do Ministério da Ciência e Ensino Superior de Portugal, desenvolve investigação na área da Mentoria Escolar e da Intervenção Sistémica em Contexto Escolar.

Madalena Alarcão é licenciada e doutorada em Psicologia pela Universidade de Coimbra, professora associada da Faculdade de Psicologia e Ciências de Educação e terapeuta familiar. Leciona na área da Família e Intervenção Sistémica; investiga na área da família, rede social e processo terapêutico e supervisiona projetos de investigação e intervenção nas áreas: Educação Parental, Mentoria Escolar, Avaliação e Intervenção com Famílias Multidesafiadas. 
